\title{
Increased p190RhoGEF expression in activated B cells correlates with the induction of the plasma cell differentiation
}

\author{
Yun Jung $\mathrm{Ha}^{1}$, Ji Hye Jeong ${ }^{1}$, Yuna Park ${ }^{1}$ \\ and Jong Ran Lee $e^{1,2,3,4}$ \\ ${ }^{1}$ Division of Life and Pharmaceutical Sciences \\ ${ }^{2}$ Department of Life Science \\ College of Natural Sciences \\ ${ }^{3}$ Center for Cell Signaling and Drug Discovery Research \\ Ewha Womans University \\ Seoul 120-750, Korea \\ ${ }^{4}$ Corresponding author: Tel, 82-2-3277-3762; \\ Fax, 82-2-3277-3760; E-mail, jrlee@ewha.ac.kr \\ http://dx.doi.org/10.3858/emm.2012.44.2.009
}

Accepted 22 November 2011

Available Online 23 November 2011

Abbreviations: AID, activation-induced deaminase; Bcl-6, B-cell lymphoma protein-6; Blimp-1, B lymphocyte-induced maturation protein-1; $\mathrm{CA}$, constitutively active; $\mathrm{DN}$, dominant negative; EV, empty vector; GC, germinal center; HA, hemagglutinin; IB, immunoblot; IRF-4, interferon regulatory factor-4; MFI, mean fluorescence intensities; Pax5, paired-box-protein 5; PC, plasma cell; PE, phycoerythrin; PerCP, peridinin chlorophyll protein; p190RhoGEF, p190 Rho guanine nucleotide exchange factor; XBP-1, X-box-binding protein-1

\begin{abstract}
Previously, we demonstrated that the p190 Rho guanine nucleotide exchange factor (p190RhoGEF) was induced following CD40 stimulation of $B$ cells. In this study, we examined whether p190RhoGEF and a downstream effector molecule RhoA are required for $B$ cell differentiation. Expression of p190RhoGEF positively correlated with the expression of surface markers and transcriptional regulators that are characteristic of mature B cells with plasma cell (PC) phenotypes. Moreover, either the overexpression of p190RhoGEF or the expression of a constitutively active RhoA drove cellular differentiation toward PC phenotypes. B cell maturation was abrogated in cells that overexpressed p190RhoGEF and a dominant-negative form of RhoA simultaneously. CD40-mediated maturation events were also abrogated in cells that overexpressed either
\end{abstract}

dominant-negative p190RhoGEF or RhoA. Together, these data provide evidence that p190RhoGEF signaling through RhoA in CD40-activated B cells drives the induction of the PC differentiation.

Keywords: antigens, CD40; ARHGAP35 protein, human; B-lymphocytes; CD40; cell differentiation; p190RhoGEF; plasma cells; rhoA GTP-binding protein

\section{Introduction}

Many of the signaling molecules and transcription factors that regulate $B$ cell fate decisions have been well characterized. Key transcriptional regulators, including $B$ lymphocyte-induced maturation protein-1 (Blimp-1), X-box-binding protein-1 (XBP-1), and interferon regulatory factor-4 (IRF-4), are required for plasma cell (PC) differentiation and function (Calame, 2001; Shapiro-Shelef and Calame, 2005). Blimp-1 is a major regulator of PC differentiation and generation of Ab-secreting cells (Turner et al., 1994; Shaffer et al., 2002, 2004; Shapiro- Shelef et al., 2003). Blimp-1 suppresses the transcriptional repressor paired- box-protein 5 (Pax5), which is essential for the commitment of lymphoid progenitors to the $B$ cell lineage, and also functions in late $B$ cell development and activation by inducing activation-induced deaminase (AID) expression (Delogu et al., 2006; Klein et al., 2006; Nera et al., 2006). AID, a novel member of the RNA editing cytidine deaminase family, is specifically expressed in germinal center (GC) B cells and is essential for somatic hypermutation and isotype switching/class switch recombination (Gray et al., 1994; Muramatsu et al., 2000; Xu et al., 2007). Blimp-1 suppresses another transcriptional repressor, B cell lymphoma protein-6 (Bcl-6), a major regulator of GC B cells (Ye et al., 1997; Delogu et al., 2006; Nera et al., 2006). Blimp-1 is thought to act upstream of XBP-1, a transcriptional regulator of the secretory pathway in PC (Reimold et al., 2001). Recently, IRF-4 has also been identified as a second major gene in PC differentiation (Klein et al., 2006; Sciammas et al., 2006). 
A

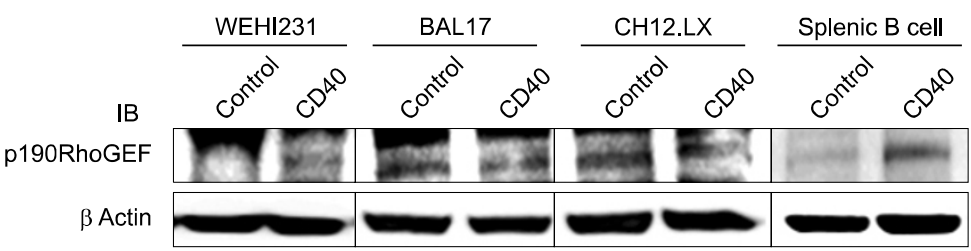

B

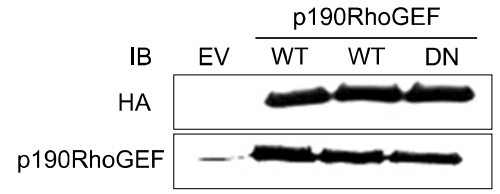

D

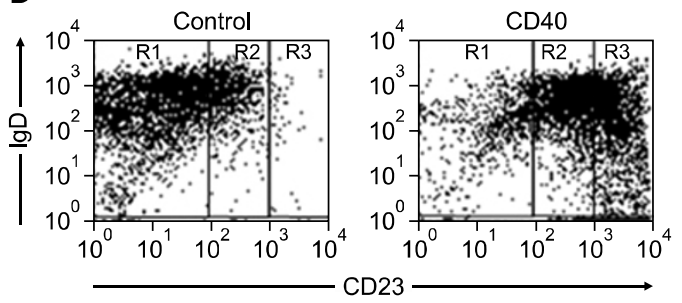

C

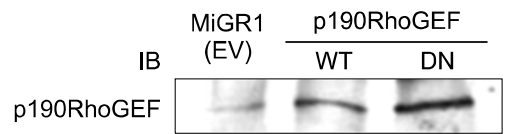

E

$\%$
population

\begin{tabular}{cr}
\multicolumn{2}{c}{ MFI } \\
p190RhoGEF \\
\hline Control & CD40 \\
\hline 13.31 & 9.68 \\
11.81 & 15.53 \\
35.58 & 50.75 \\
\hline
\end{tabular}

Figure 1. p190RhoGEF protein levels and changes after CD40 stimulation of $B$ cells of various maturational stages. (A) Mouse splenic $B$ cells $\left(1 \times 10^{6} / \mathrm{ml}\right)$ and the B cell lines WEHI 231, BAL17, CH12.LX $\left(5 \times 10^{5} / \mathrm{ml}\right)$ were stimulated with isotype control lg or anti-CD40 at a concentration of $1 \mu \mathrm{g} / \mathrm{ml}$ for $48 \mathrm{~h}$. (B) HEK 293T cells were transfected with plasmids carrying an empty vector (EV), WT, or DN p190RhoGEF (HA). (C) WEHI 231 B cells were stably transfected with MIGR1 (EV), WT, or DN p190RhoGEF. Lysates were prepared from these cells, subjected to a SDS-PAGE (6\% or $8 \%$ gels), transferred to nitrocellulose, and probed with an Ab specific for p190RhoGEF (A-C) or HA (B). Equal loading of the protein samples was demonstrated by anti- $\beta$ actin IB in $(A)$. (D, E) Mouse splenic B cells $\left(1 \times 10^{6} / \mathrm{ml}\right)$ were stimulated with control lg or anti-CD40 at a concentration of $1 \mu \mathrm{g} / \mathrm{ml}$. After $48 \mathrm{~h}$ of stimulation, cells were fixed, permeabilized, and stained for endogenous p190RhoGEF, CD23, and IgD. The fluorescence intensities of cells stained with an isotype control $\mathrm{Ab}$ or antiserum were all corrected (data not shown). The results from flow cytometry are presented as a dot plot, showing lgD versus CD23 positive cells. For the cell populations in R1, R2, and R3 as indicated in (D), the population of cells (\%) and the expression level of p190RhoGEF as mean fluorescence intensity (MFI) in the absence (control) and presence of CD40 stimulation is presented in (E). The results shown are representative of three independent experiments.

CD40 is an important cell surface receptor for Ag-dependent and $\mathrm{T}$ helper cell-regulated terminal differentiation of naïve $B$ cells into Ab-secreting plasma and memory cells. The molecular mechanisms underlying the developmental coordination of CD40 stimulation with lg secretion has been well characterized by many groups (Gray et al., 1994; Kawabe et al., 1994; Benson et al., 2007). However, the context in which CD40-CD154 signaling acts to activate transcription factors that regulate $B$ cell fate decisions is not clearly understood.

Previously, we identified the p190 Rho guanine nucleotide exchange factor ( $p 190 \mathrm{RhoGEF}$ ) as a protein that is strongly induced in response to CD40 stimulation of B cells (Lee et al., 2003). We also demonstrated that the expression of p190RhoGEF is correlated with changes in cellular structure following CD40 stimulation in WEHI 231 B cells (Lee et al., 2003). Furthermore, cells that transiently overexpressed p190RhoGEF behaved as if they expressed a constitutively active (CA) form of RhoA (Q63L), whereas transient overexpression of dominant negative (DN) forms of p190RhoGEF (Y1003A) or RhoA (T19N) blocked the CD40-mediated activation effects (Lee et al., 2003). These results suggest that there may exist a relationship between p190RhoGEF, the morphological changes in CD40-activated B cells, and additional B cell maturation and differentiation events.

In the present study, we examined whether p190RhoGEF expression is correlated with the expression of specific surface markers and transcriptional regulators that are characteristic of both mature and differentiated $B$ cells. We also examined whether the activities of p190RhoGEF and RhoA are required for CD40-mediated $B$ cell maturation and differentiation. Our results demonstrate that the expression of p190RhoGEF correlates with the expression of the cell surface markers CD138 (syndecan-1) and CXCR4, as well as the transcription factors Blimp-1, XBP-1, and IRF-4. The expression of these molecules was also induced following CD40 stimulation, as well as by overexpressing either wild type (WT) p190RhoGEF or a 
A
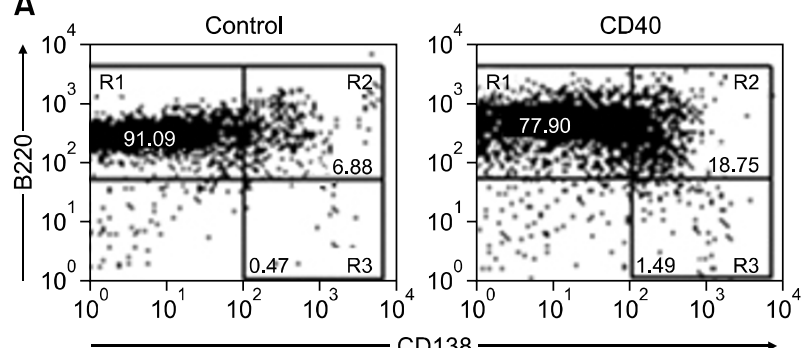

B

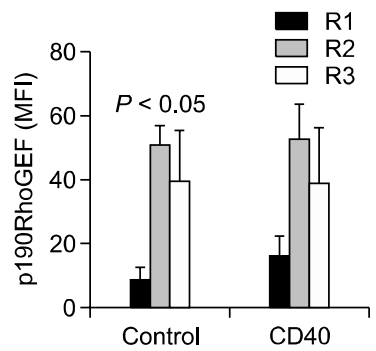

C
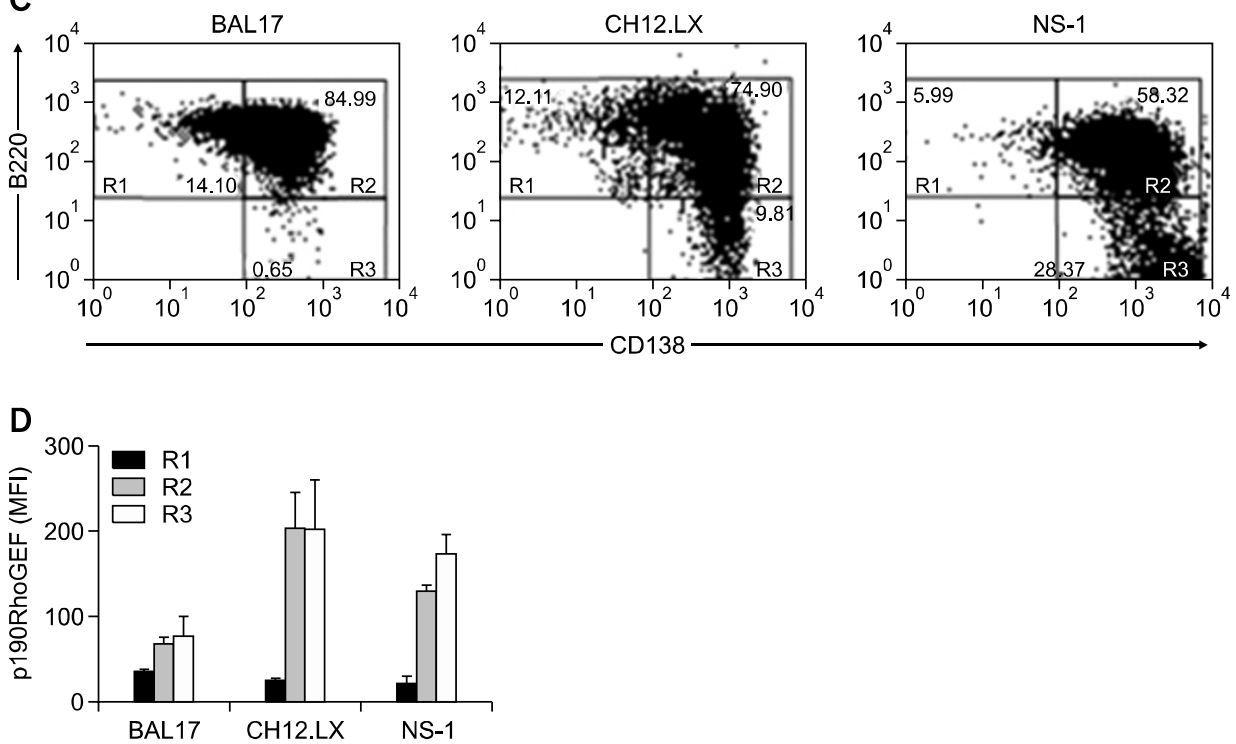

Figure 2. Expression of p190RhoGEF, CD138, and B220 in B cells at various maturational stages. (A, B) Mouse splenic B cells $\left(1 \times 10^{6} / \mathrm{ml}\right)$ were stimulated with control $\mathrm{lg}$ or anti-CD40 at a concentration of $1 \mu \mathrm{g} / \mathrm{ml}$. After $72 \mathrm{~h}$ of stimulation, cells were fixed, permeabilized, and stained for endogenous p190RhoGEF, B220, and CD138. The fluorescence intensities of cells stained with an isotype control Ab or antiserum were all corrected (data not shown). The results from flow cytometry are presented as a dot plot, showing the B220 positive cells versus the CD138 positive cells. The data shown are representative of three independent experiments. For the cell populations indicated in (A), expression of p190RhoGEF is presented as MFI in (B). Data are presented as the mean $(n=4) \pm$ SD. (C, D) The B cell lines, BAL17, CH12.LX, and NS-1 were fixed, permeabilized, and stained for endogenous p190RhoGEF, CD138, and B220. The results were obtained as described above. For the cell populations indicated in (C), expression of p190RhoGEF is presented as MFI in (D). Data are presented as the mean $(n=3) \pm$ SD.

CA form of RhoA (Q63L). Collectively, these data suggest a specific contribution of p190RhoGEF to CD40-mediated differentiation and that $190 \mathrm{RhoGEF}$ may be a crucial component of $T$ cell-dependent $B$ cell maturation and differentiation.

\section{Results}

\section{Changes in expression of surface markers and p190RhoGEF by CD40 stimulation}

We previously reported that a $48 \mathrm{~h}$ stimulation of CD40 leads to increases in p190RhoGEF expression and causes characteristic structural changes in $B$ cells (Lee et al., 2003). Because the cellular structure of $B$ cells changes during cell maturation, we tested whether p190RhoGEF expression corre- lates with stages in the maturation of $B$ cells following CD40 stimulation. Analysis of the protein levels of $p 190 R$ hoGEF in B cells of various maturational stages also demonstrated that there was an increase in p190RhoGEF protein levels following CD40 stimulation of WEHI 231 and splenic B cells, but not the mature $B$ cell lines, BAL17 and CH12.LX (Figure 1A). The specificity of the polyclonal Ab generated against p190RhoGEF was characterized in HEK 293T cells transfected with hemagglutinin (HA)-p190RhoGEF expression plasmids (Figure 1B) and in WEHI 231 B cells in which p190RhoGEF was overexpressed (Figure 1C).

As $B$ cell maturation progresses, expression of $\mathrm{CD} 23$ is increased and expression of $\operatorname{lgD}$ is decreased. We further analyzed expression of the surface markers IgD and CD23. We used flow cy- 
tometry to analyze whether CD40 stimulation caused changes in the expression of $\operatorname{lgD}$ and CD23. Changes in the expression level of $\lg D$ versus CD23 in mouse splenic B cells treated with anti-CD40 are shown in Figure 1D. B cell population was defined as three groups (R1: $C D 23^{\text {low }}, \mathrm{R} 2$ : CD23 ${ }^{\text {medium }}$, R3: CD23 ${ }^{\text {low }}$ ) based on the level of CD23 expression. Results in Figure 1D and 1E demonstrate that CD40 stimulation of these B cells results in a change in maturation stage, indicated as a percentage of cell population in R1-R3. p190RhoGEF expression correlates to changes in cellular maturation both in unstimulated control and CD40 stimulation of splenic $B$ cells (Figure 1E).

\section{Expression of p190RhoGEF is correlated with the expression of CD138 and transcriptional regulators driving PC differentiation}

To determine whether $\mathrm{p} 190 \mathrm{Rh}$ oGEF expression induced by CD40-stimulated B cell activation is involved in $\mathrm{PC}$ differentiation, we correlated the expression of $p 190$ RhoGEF with the expression of a specific surface molecule, CD138, which has been previously implicated as a PC marker (Calame, 2001; Shapiro-Shelef and Calame, 2005). B cell population was separated by the expression level of B220 and CD138 (R1: B220 ${ }^{\text {high }}$ CD138 ${ }^{\text {low }}$, R2: $\mathrm{B} 220^{\text {high }} \mathrm{CD} 138^{\text {high }}$, R3: B220 $\left.{ }^{\text {low }} \mathrm{CD} 138^{\text {high }}\right)$. By flow cytometry, we found that following $72 \mathrm{~h}$ of CD40 stimulation, the $B$ cell population expressing CD138 on the surface increased from $-7.4 \%$ to $-20 \%$ (R2 and R3) in mouse splenic $B$ cells (Figure 2A). Accordingly, the expression of p190RhoGEF was analyzed in the regions (R1, R2, and R3) separated by the expression level of $\mathrm{B} 220$ and CD138. As shown in Figure 2B, p190RhoGEF expression increased in R2 and R3 in mouse splenic $B$ cells over the expression level in R1 both in unstimulated controls and following CD40 stimulation. CD40 stimulation also increased p190RhoGEF expression -2-fold in R1compared to the level of unstimulated controls.

To directly correlate the expression of p190RhoGEF with PC differentiation, we analyzed the expression levels of B220 versus CD138 in the mature $B$ cell lines with PC phenotypes, BAL17 and $\mathrm{CH} 12 . \mathrm{LX}$, and in plasmacytoma NS-1 cells. As shown in Figure $2 \mathrm{C}$, populations demonstrating distinct maturation stages differ slightly when R1-R3 are compared. Further analysis of the expression of $\mathrm{p} 190 \mathrm{RhoGEF}$ in these cell populations demonstrated that p190RhoGEF expression is much higher in cells that show more characteristics of PC with higher population in R3 $(\mathrm{CH} 12 . \mathrm{LX}$ and NS-1 cells with higher population in R3 vs. BAL17
A

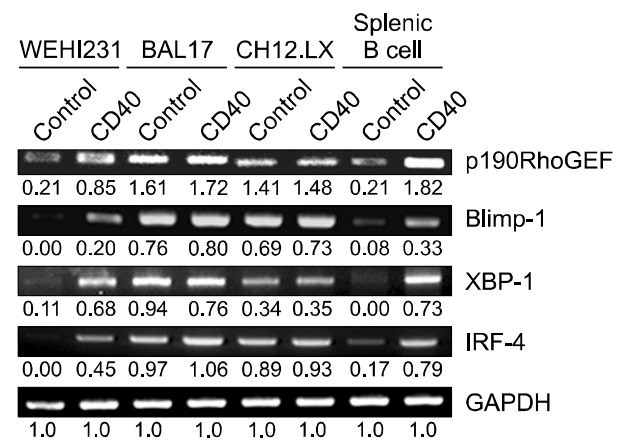

B

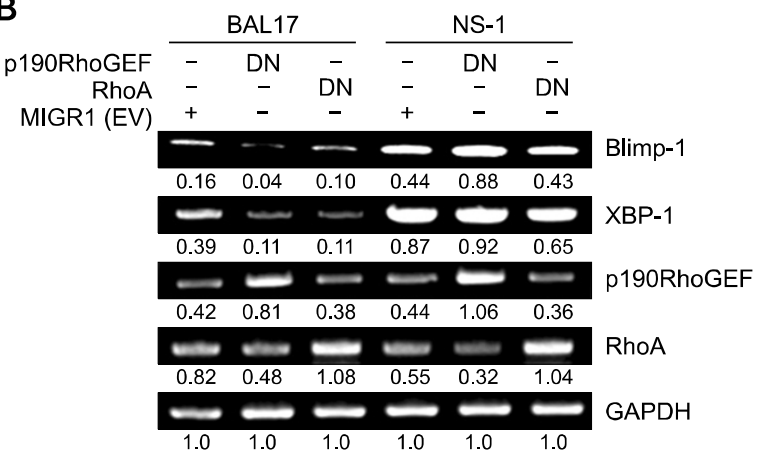

Figure 3. Expression changes of transcriptional regulators after $\mathrm{CD} 40$ stimulation and in response to p190RhoGEF and RhoA down-regulation in $B$ cells at various maturational stages. (A) $c D N A$ was prepared from $B$ cells that were either untreated (control) or stimulated for $24 \mathrm{~h}$ with anti-CD40 $(1 \mu \mathrm{g} / \mathrm{mL})$. (B) cDNA was prepared from BAL17 and NS-1 cells that were stably transfected with MIGR1 (EV), p190RhoGEF (Y1003A) or RhoA (T19N). The PCR products for p190RhoGEF, RhoA, Blimp-1, XBP-1, IRF-4, and GAPDH were separated as described in "Methods." The data shown are representative of three independent experiments. Values indicate normalization for GAPDH.

cells with lower population in R3 (Figure 2D).

Because the transcriptional regulators, Blimp-1, $\mathrm{XBP}-1$, and IRF-4 are known to play key roles in PC development (Turner et al., 1994; Reimold et al., 2001; Shaffer et al., 2002; Shapiro-Shelef et al., 2003; Shaffer et al., 2004; Klein et al., 2006; Sciammas et al., 2006), we next examined whether p190RhoGEF expression also correlated with the expression of these transcriptional regulators. The expression of Blimp-1, XBP-1, and IRF-4 was compared with the CD40-induced changes in these regulators compared to the unstimulated controls. As shown in Figure 3A, CD40 stimulation greatly enhanced the expression of these transcripts in WEHI 231 and splenic B cells. In contrast, the expression of Blimp-1, XBP-1, and IRF-4 is high in BAL17 and CH12.LX cells, independent of CD40 stimulation (Figure $3 \mathrm{~A}$ ).

Similarly, p190RhoGEF expression was constitutively high in BAL17 and $\mathrm{CH} 12 . \mathrm{LX}$ cells, but was induced in WEHI 231 and splenic B cells after 
A

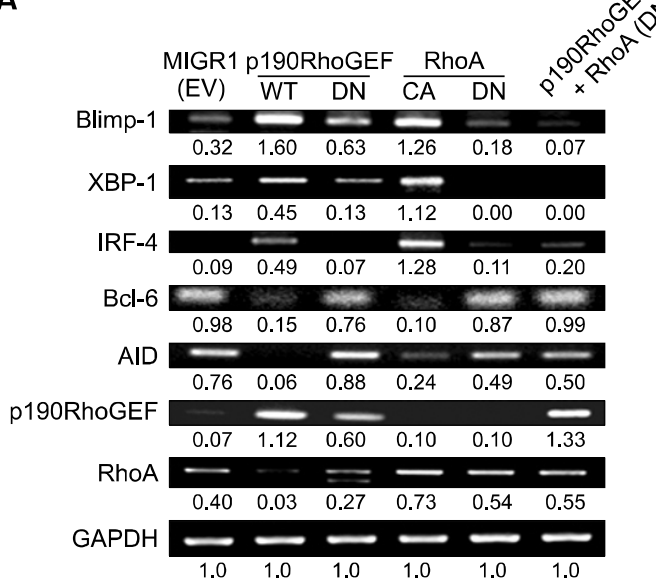

C

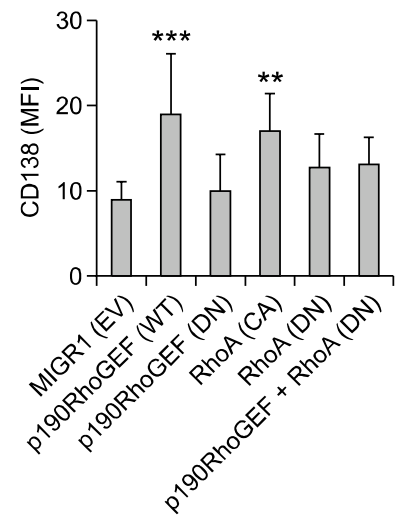

B

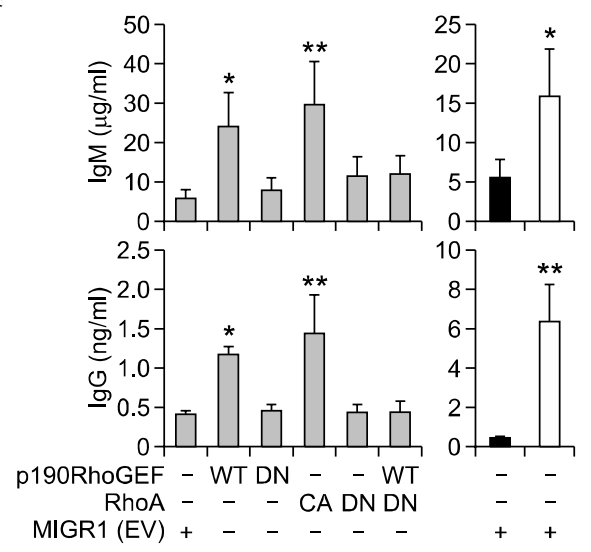

D

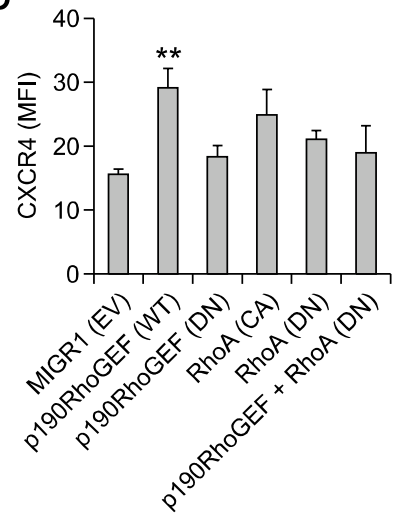

Figure 4. Direct effects of manipulating the activity of p190RhoGEF and RhoA on B cell maturation and downstream transcriptional regulators. WEHI 231 B cells were stably transfected with MIGR1 (EV), WT p190RhoGEF, DN p190RhoGEF (Y1003A), CA RhoA (Q63L), DN RhoA (T19N), or a combination of WT p190RhoGEF and DN RhoA (T19N). (A) CDNA was prepared from these stably transfected cells. The PCR products for p190RhoGEF, RhoA, Blimp-1, XBP-1, IRF-4, BCl-6, AID, and GAPDH were separated as described in "Methods." The data shown are representative of three independent experiments. Values indicate normalization for GAPDH. (B) These stably transfected cells were removed from the cultures and then washed, counted, and recultured in fresh medium for $24 \mathrm{~h}$ at $1 \times 10^{6}$ cells $/ \mathrm{ml}$. Some EV-transfected cells were also incubated with a medium alone (black bars) or with a combination of CD40 and IL4 as positive controls (white bars). Supernatants were harvested and serially diluted, and secreted Ig was measured by anti-lgM and anti-IgG ELISA as described in "Methods." Values of the optical densities at $490 \mathrm{~nm}$ in triplicate supernatant samples were collected in each independent experiment. Data are presented as the mean of three independent experiments $(n=3) \pm \mathrm{SD},{ }^{*} P<0.05,{ }^{* *} P<0.01$, compared with cells transfected with EV. (C, D) These stably transfected cells were fixed, stained with anti-CD138 or anti-CXCR4, and analyzed by standard flow cytometry. The fluorescence intensities of cells stained with an isotype control Ab or antiserum were all corrected (data not shown). The bar graphs show the expression of CD138 (C) or CXCR4 (D), as indicated by MFI. Data are presented as the mean of three independent experiments $(n=3) \pm$ SEM, ${ }^{* *} P<0.01$, ${ }^{* * *} P<$ 0.001 , compared with cells transfected with EV.

CD40 stimulation (Figures $1 \mathrm{~A}$ and $3 \mathrm{~A}$ ). These results strongly suggest that the expression of p190RhoGEF mediates the induction of key transcriptional regulators, Blimp-1, XBP-1, and IRF-4, which regulate $B$ cell maturation and differentiation.

Because p190RhoGEF specifically activates RhoA (Gebbink et al., 1997; van Horck et al., 2001), we next examined whether the activities of p190RhoGEF and its downstream effector RhoA are required during $P C$ differentiation. As shown in Figure $3 \mathrm{~B}$, the expression levels of the major transcriptional regulators of $\mathrm{PC}$ maturation and differentiation, Blimp-1 and XBP-1 were greatly reduced in BAL17 cells transfected with a DN form of p190RhoGEF (Y1003A) or RhoA (T19N). These results strongly suggest that one function of p190RhoGEF is to maintain the expression of key transcriptional regulators of $\mathrm{B}$ cell maturation and differentiation. In contrast, the DN form of either p190RhoGEF or RhoA had no effect on the expression of these transcription factors in NS-1 cells (Figure 3B). These results also suggest that p190RhoGEF functions in maturing $B$ cells, but not in completely differentiated plasmacytoma cells. 


\section{Expression of p190RhoGEF and activation of RhoA mimic CD40-induced $B$ cell maturation and differentiation}

We next determined whether p190RhoGEF expression induced by CD40-stimulated B cell activation is involved in the PC differentiation process. To determine the requirement for $\mathrm{p} 190 \mathrm{RhoGEF}$ in expression of the key transcriptional regulators Blimp-1, XBP-1, and IRF-4, we transfected WEHI 231 B cells with a plasmid encoding a WT or DN form of p190RhoGEF and measured the subsequent expression of these regulators. We found that the expression of these transcription factors increased in cells transfected with WT p190RhoGEF, as well as a CA RhoA (Q63L), compared to cells transfected with the DN form of the proteins (Figure 4A). The expression of Blimp-1, XBP-1, and IRF-4 was not induced in cells that simultaneously expressed the WT p190RhoGEF and DN RhoA (T19N) (Figure 4A). Induced expression of Blimp-1 in WEHI 231 B cells transfected with WT p190RhoGEF or CA RhoA (Q63L) stimulated these cells to become Ab-secreting cells by suppressing Bcl- 6 and AID expression (Figure 4A). Secretion of $\lg M$ and $\lg G$ $A b$ was detected high in the supernatants of these cells transfected with the WT p190RhoGEF as well as CA RhoA (Q63L) (Figure 4B). Some transcriptional increase shown in cells transfected with $D N$ forms of p190RhoGEF and RhoA could possibly be due to controlled regulation by overexpression of these plasmids.

Similarly, we found that expression of the B cell surface differentiation markers CD138 and CXCR4 increased significantly in cells transfected with WT p190RhoGEF, but expression was unchanged in cells transfected with the DN plasmid (Figures $4 \mathrm{C}$ and 4D). We also found that these surface markers were upregulated in cells transfected with $C A$ RhoA (Q63L) but not much in cells transfected with the DN form of RhoA (T19N) (Figures 4C and 4D). Moreover, the expression of surface markers in cells that simultaneously expressed DN RhoA (T19N) and WT p190RhoGEF was not induced, confirming that RhoA acts downstream of p190RhoGEF (Figures 4C and 4D).

\section{The p190RhoGEF-RhoA activation is required for the CD40-induced $B$ cell differentiation}

We also tested to see whether $\mathrm{p} 190 \mathrm{RhoGEF}-\mathrm{RhoA}$ activation is required for CD40-induced expression of surface molecules. In cells that were transfected with a vector plasmid, CD40 stimulation induced the expression of cell surface molecules (Figures $5 \mathrm{~A}$ and $5 \mathrm{~B}$ ). However, in cells that were transfected with a DN form of either p190RhoGEF (Y1003A) or
A

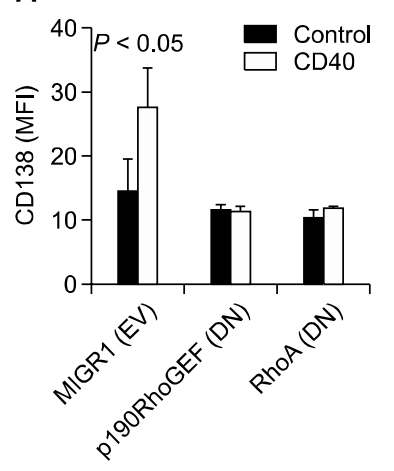

B

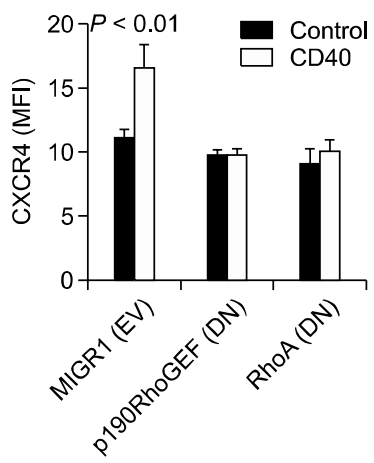

C

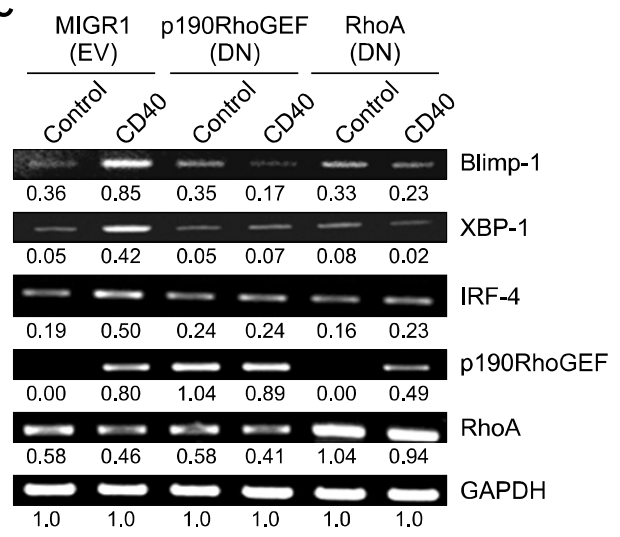

Figure 5. Effects of the overexpressed DN forms of p190RhoGEF or RhoA on the expression of CD138, CXCR4, and transcriptional regulators after CD40 stimulation. WEHI $231 \mathrm{~B}$ cells were stably transfected with either MIGR1 (EV), a plasmid encoding DN p190RhoGEF (Y1003A), or a plasmid encoding DN RhoA (T19N). (A, B) After CD40 stimulation for $48 \mathrm{~h}$, cells were fixed, stained with anti-CD138 or anti-CXCR4, and measured by flow cytometry. The fluorescence intensities of cells stained with an isotype control $A b$ or antiserum were all corrected (data not shown). The results show the expression intensities of CD138 (A) or CXCR4 (B). Data are presented as the mean of three independent experiments $(n=3) \pm$ SD. (C) cDNA was prepared from these stably transfected cells, after being stimulated for $48 \mathrm{~h}$ with anti-CD40 Ab (1 $\mu \mathrm{g} / \mathrm{ml})$. The PCR products for p190RhoGEF, RhoA, Blimp-1, XBP-1, IRF-4, and GAPDH were separated as described in "Methods." The data shown are representative of three independent experiments. Values indicate normalization for GAPDH.

RhoA (T19N), CD40 stimulation had no effect on cell surface protein levels (Figures $5 \mathrm{~A}$ and $5 \mathrm{~B}$ ).

Additionally, CD40 stimulation had no effect on the expression of transcriptional regulators in cells that were transfected with a $D N$ form of either p190RhoGEF (Y1003A) or RhoA (T19N). However, in RhoA (T19N)-transfected WEHI 231 cells, p190RhoGEF induction was shown (Figure 5C). In cells that were transfected with a vector plasmid, CD40 stimulation induced the expression of p190RhoGEF, but had little effect on RhoA expression levels (Figure $5 \mathrm{C}$ ). These results strongly suggest that the enhanced activation of RhoA is 
mediated by $\mathrm{p} 190 \mathrm{RhoGEF}$ after CD40 stimulation in WEHI 231 B cells.

\section{Discussion}

CD40 is an important surface receptor that is involved in the activation and maturation of $B$ cells during immune responses (Foy et al., 1994; Gray et al., 1994; Kawabe et al., 1994; Xu et al., 1994; Arpin et al., 1995; Randall et al., 1998; Yoon et al., 2005). CD40-CD154 interactions are crucial for the initiation and propagation of GC reactions following exposure to exogenous $\mathrm{Ag}$ and for the production of high affinity, class-switched Ab. However, the role of CD40 signaling in B cell differentiation is controversial (Foy et al., 1994; Gray et al., 1994; Kawabe et al., 1994; Xu et al., 1994; Arpin et al., 1995; Randall et al., 1998). Earlier studies have indicated that the stimulation of $B$ cells through CD40 leads to the development of memory B cells and not PCs (Arpin et al., 1995). Studies have also demonstrated that the interaction of CD40 with its cell surface ligand CD154 actively suppresses Ig secretion in B cells and eventually allows for the selection of high affinity Ig-producing cells in the GC (Randall et al., 1998). In contrast, other reports have suggested that signaling through CD40 promotes the terminal differentiation of $B$ cells into PCs and Ig secretion, particularly in combination with cytokines (Foy et al., 1994; Gray et al., 1994; Xu et al., 1994). Several studies have also demonstrated CD40-dependent, T cell-dependent, humoral immunity through the disruption of genes involved in CD40 signaling pathways (Ahonen et al., 2002; Jabara et al., 2002; Gallagher et al., 2007). Here, we have shown that CD40 signaling through p190RhoGEF induces expression of the transcription factors Blimp-1, XBP-1, and IRF-4, as well as the cell surface proteins CD138 and CXCR-4, which drive cells to become Ab-secreting cells.

p190RhoGEF, which was cloned and identified as a RhoA specific GEF (Gebbink et al., 1997; van Horck et al., 2001), has not been reported to function in immune cells. We previously reported that expression of p190RhoGEF is markedly enhanced after CD40 stimulation in WEHI 231 B cells and is correlated with cellular structural changes and NF-kB activation (Lee et al., 2003). These results may indicate that this protein plays a significant role in CD40-mediated $B$ cell activation and maturation. Here, we investigated the specific contribution of p190RhoGEF in CD40-stimulated cells in order to further our understanding of the molecular mechanisms underlying $B$ cell maturation and differentiation.
Ag-dependent terminal differentiation of naïve B cells requires $T$ helper cell regulation for cell expansion and effector and memory cell development. Recently, naïve $B$ cell priming, which is central to the initiation phase of $T$ cell-dependent $B$ cell responses, has been studied extensively (Gray, 2002; McHeyzer-Williams and McHeyzer-Williams, 2005). The transcription factor Blimp-1 has been shown to be both necessary and sufficient for the differentiation of naïve B cells into PCs (Turner et al., 1994; Shaffer et al., 2002, 2004; Shapiro-Shelef et al., 2003). Deletion of the transcription factor Pax5, which is continuously required to maintain $B$ cell identity by alternate lineage genes, results in the down-regulation of the GC transcriptional regulator $\mathrm{Bcl}-6$, which consequently induces expression of the PC transcription factors Blimp-1 and XBP-1 (Ye et al., 1997; Reimold et al., 2001; Delogu et al., 2006; Nera et al., 2006). In an activated GC B cell state, physiological levels of Blimp-1 expression are induced by the down-regulation of Pax5 as well as by positive regulatory inputs from the $\mathrm{Ag}$ and cytokine receptor signaling systems (Delogu et al., 2006; Nera et al., 2006). Although recent studies have begun to dissect cellular events within the GC (Kallies et al., 2007), there are still many unanswered questions as to how PCs become terminally differentiated.

Our findings suggest that the strong stimulation of $B$ cells by activated T cells (through CD40-CD154 interactions), induces Blimp-1 expression and differentiation into $B$ cells with $P C$ phenotype. The expression levels of $p 190$ RhoGEF are low in splenic $B$ cells as well as in an immature $B$ cell line, WEHI 231, but increase following CD40 activation (Figures $1 \mathrm{~A}$ and $4 \mathrm{~A})$. In contrast, elevated $\mathrm{p} 190 \mathrm{RhoGEF}$ expression levels do not increase further following CD40 activation in mature $B$ cells that have a PC phenotype (e.g., BAL17 and CH12.LX) (Figures 1A and $4 A$ ). CD40 activation induces a change in $B$ cell maturation as well as in p190RhoGEF expression levels (Figure 1D). The expression levels of $p 190$ RhoGEF increase in mature $B$ cells with $P C$ phenotypes (Figure 2). The expression of key transcription factors required for $\mathrm{PC}$ differentiation (Blimp-1, XBP-1, and IRF-4) was also upregulated by CD40 activation (Figure $3 A$ ). Overexpression of the DN form of $p 190$ RhoGEF or RhoA in mature BAL-17 B lymphoma cells significantly diminished the level of these transcriptional regulators (Figure $3 B$ ). Interestingly, these negative regulatory effects on $B$ cell maturation were not seen in experiments where p190RhoGEF activity was blocked in plasmacytoma cells, which are terminally differentiated (Figure 3B). Moreover, overexpression of the WT or DN forms of p190RhoGEF or RhoA directly 
modulated the expression of these transcription factors in the absence of CD40 stimulation in WEHI 231 B cells (Figure 4A). Secretion of IgM and $\lg G A b$ in the supernatants of cells transfected with the WT p190RhoGEF as well as CA RhoA (Q63L) (Figure 4B) suggest that $p 190$ RhoGEF also regulates AID expression in the GC reaction before turning on the expression of Blimp-1, which induces $P C$ differentiation. Our results indicate that p190RhoGEF is induced in activated $B$ cells by CD40 and is required for initial triggering of GC B cell differentiation into cells of PC phenotype.

The Rho family of GTPases has been shown to regulate cell polarization, motility, and chemotaxis in many cell types (Ridley, 2001; Boettner and Aelst, 2002; Etienne-Manneville and Hall, 2002; Meili and Firtel, 2003). Specifically, RhoA regulates the maturation of focal complexes into focal adhesions and the formation of associated stress fibers (Rottner et al., 1999; Ridley, 2001; Burridge and Wennerberg, 2004). Our previous study demonstrated that the structural changes following CD40 stimulation of $B$ cells was due to p190RhoGEF-induced RhoA activity (Lee et al., 2003). Those results are consistent with the findings from this investigation which show that changes in cellular morphology are regulated by $\mathrm{p} 190 \mathrm{RhoGEF}$ during CD40-mediated $\mathrm{B}$ cell activation and are correlated with Blimp-1 expression and B cell differentiation. Recent studies in macrophages have also shown that $\mathrm{Bcl}-6$ alters macrophage morphology and motility by suppressing RhoA activity (Pixley et al., 2005). In this respect, a number of recent studies have highlighted the importance of the cytoskeleton in various lymphocytic functions including migration, Ag recognition, signaling, and activation (Vicente-Manzanares and Sanchez-Madrid, 2004). Thus, additional studies are needed to determine whether cytoskeletal reorganization during CD40mediated $B$ cell activation contributes to GC B cell differentiation into cells of PC phenotype.

Recent studies have provided evidence that RhoA is involved in cell signaling and directly controls gene transcription and cell cycle progression (Li et al., 2000; Gu et al., 2003, 2005; Walmsley et al., 2003). Genetic studies have shown that Rho family members are required for normal development and functioning of lymphocytes ( $\mathrm{Li}$ et al., 2000; Gu et al., 2003, 2005; Walmsley et al., 2003). RhoA has also been implicated in regulating $B$ cell receptor-dependent calcium flux and proliferation (Saci and Carpenter, 2005), as well as Wnt-mediated migration and invasion of myeloma PCs (Qiang et al., 2005). Here, we provide evidence that RhoA activation is required for $B$ cell maturation by $C D 40$ receptor signaling. However, the mechanism by which CD40-mediated p190RhoGEFRhoA activation drives the induction of transcriptional regulators, including Blimp-1 and XBP-1, remains to be determined.

In summary, this report shows that CD40 signaling induces p190RhoGEF expression, which in turn activates RhoA and results in the expression of surface molecules and transcriptional regulators that are required for the maturation and differentiation of $B$ cells. We have demonstrated a strong correlation between the expression levels of p190RhoGEF, the cell surface markers syndecan-1 and CXCR4, and the transcriptional regulators Blimp-1 and XBP-1. These data implicate RhoA and p190RhoGEF as crucial components mediating $T$ cell-dependent $B$ cell maturation and differentiation.

\section{Methods}

\section{$\mathrm{Ab}$ and reagents}

The rat anti-mouse CD40 mAb (clone 1C10) from R\&D Systems, Inc. (Minneapolis, MN) and the hamster anti-mouse CD40 mAb (HM40-3) from BD PharMingen (San Diego, $C A$ ) were used to stimulate $B$ cells. To isolate splenic $B$ cells, the B cell isolation kit (Miltenyi Biotec, Bergisch Giadbach, Germany) was used for depletion of non-B cells (negative selection). For cell surface staining, r-phycoerythrin (PE)-conjugated rat anti-mouse CD138 (syndecan-1; 281-2), CXCR4 (2B11), CD23 (B3B4), and peridinin chlorophyll protein (PerCP)-conjugated rat anti-mouse CD45R/ B220 (RA-6B2) mAb were purchased from BD PharMingen. Biotin-conjugated rat anti-mouse IgD (11-26) was from Southern Biotechnology Associates (Birmingham, $A L)$. FITC-conjugated goat anti-rabbit lgG, allophycocyanin- or PerCP-conjugated streptavidin, and isotype control Ig's were purchased from Jackson ImmunoResearch Laboratories, Inc. (West Grove, PA) or BD PharMingen. To stain and immunoblot (IB) p190RhoGEF, we generated a rabbit polyclonal LF-r-gef Ab using purified GST-p190 RhoGEF (aa 1424-1615) as the immunogen (Lab Frontier, Seoul, Korea) (Meyer et al., 1999). An anti-HA mouse mAb (Sigma-Aldrich, St. Louis, MO) and an anti- $\beta$-actin (Santa Cruz Biotechnology, Inc., Santa Cruz, CA) were used for IB analysis. A HRP-conjugated anti-rabbit or anti-mouse IgG (Bio-Rad, Hercules, CA) was used as a secondary Ab for IB.

\section{Plasmids}

The expression plasmids for WT and DN (Y1003A) p190RhoGEF (pCDNA3-HA) have been described previously (van Horck et al., 2001; Lee et al., 2003). To generate expression plasmids for WT and DN p190RhoGEF (MIGR1), the cDNAs encoding WT and DN p190RhoGEF were released from the pcDNA3 expression vector by digestion with Bg/ll and Notl and ligated into the pLNCX2 retroviral vector (Clontech Laboratories, Inc., Palo Alto, CA) 
before being sub-cloned into the MSCV MIGR1 bicistronic vector (a kind gift from W. Pear through G. Koretzky, University of Pennsylvania) (Pear et al., 1998), between the Bg/ll and Sall/Xhol sites. Plasmids expressing FLAGtagged CA (Q63L) and DN (T19N) RhoA (MIGR1) were generated by PCR amplification with FLAG sequence-containing primers from CA and DN RhoA (pRK5-myc) expression plasmids (kind gifts from Dr. G. M. Bokoch, The Scripps Research Institute, La Jolla, CA), followed by ligation into the MSCV MIGR1 bicistronic vector between the $B g / l l$ and EcoRI sites. Each construct was verified by sequencing.

\section{Cell culture, treatment, and isolation of splenic $B$ cells}

The mouse B lymphoma cell lines, WEHI 231, BAL17, and $\mathrm{CH} 12$. LX were cultured in B cell medium (RPMI 1640 medium supplemented with $10 \%$ heat-inactivated fetal bovine serum, $100 \mathrm{U} / \mathrm{ml}$ penicillin, $100 \mu \mathrm{g} / \mathrm{ml}$ streptomycin, $20 \mathrm{mM}$ glutamine, and $5 \times 10^{-5} \mathrm{M} 2-\mathrm{ME}$ ). A plasmacytoma cell line, NS-1, was cultured in B cell medium lacking 2-ME. The human embryonic kidney cell line HEK 293T was cultured in DMEM supplemented with $10 \%$ heat-inactivated fetal bovine serum, $100 \mathrm{U} / \mathrm{ml}$ penicillin, $100 \mu \mathrm{g} / \mathrm{ml}$ streptomycin, and $20 \mathrm{mM}$ glutamine. The cell lines were maintained at $37^{\circ} \mathrm{C}$ in $5 \% \mathrm{CO}_{2} / 95 \% \mathrm{O}_{2}$. Splenic $\mathrm{B}$ cells were isolated from adult female BALB/c mice (6 to 8 weeks old) using the $B$ cell isolation kit (Miltenyi Biotec) for depletion of non-B cells (negative selection), according to the manufacturer's instructions. When stimulated, the cells (B lymphoma, plasmacytoma, $5 \times 10^{5} / \mathrm{ml}$; splenic B cells, $1 \times$ $10^{6} / \mathrm{ml}$ ) were incubated with anti-CD40 at $1 \mu \mathrm{g} / \mathrm{ml}$ for 48 or $72 \mathrm{~h}$. The handling of animals followed institutional guidelines.

\section{Retroviral infection}

Phoenix-Ampho cells were transfected with a MIGR1 construct using the calcium phosphate precipitation method and viral supernatants were collected as described previously (Pear et al., 1993). WEHI 231, BAL17, and NS-1 cells were spin-infected with retroviral supernatants before being cultured in growth medium for 4-5 days. Fluorescent cells were separated using a FACSAria cell sorter system (BD Biosciences).

\section{Transient expression of plasmid DNA}

HEK 293T cells were seeded the day before transfection at $-6 \times 10^{6}$ cells $/ 60-\mathrm{mm}$ culture dish. The cells were then transfected with $5 \mu \mathrm{g}$ of various expression plasmids by calcium phosphate precipitation. The transfected cells were cultured in growth medium for $24 \mathrm{~h}$ before experimental manipulations.

\section{RNA isolation and RT-PCR}

Total RNA from B lymphoma cells and splenic B cells that were either unstimulated or stimulated was isolated using the TRIzol reagent that was purchased from Life Technologies (Grand Island, NY). For RT-PCR, the total
RNA was a template for cDNA synthesis with oligo (dT) as the primer. The Oligo (dT) $12-18$ primer and M-MLV-reverse transcriptase were received from Promega (Madison, WI). A pair of primers was used to amplify each desired CDNA (p190RhoGEF: 5'-gcctctagatcttctctgtggatcgac-3', 5'-tccagc agccatctaagcagg-3'; RhoA: 5'-gaagatctatggctgccatcag-3', 5'-cggaattctcacaagatgaggc-3'; Blimp-1: 5'-gccaaccaggaact tcttgtgt-3', 5'-aggataaaccacccgagggt-3'; XBP-1: 5'-gctgcgg aggaaactgaaa-3', 5'-gggaggctggtaaggaact-3'; IRF-4: 5'-gaa gatctatggctgccatcag-3', 5'-cggaattctcacaagatgaggc-3'; Bcl6: 5'-tcagagtattcggattctagctgtga-3', 5'-tgcagcgtgtgcctcttg-3'; Pax5: 5'-gcgtgtttgagagacagcact-3', 5'-aagaatactgagggtggctgt-3'; AID: 5'-ggctgaggttagggttccatctcag-3', 5'-gagggagtcaagaaa gtcacgctgga-3'; GAPDH: 5'-atcaccatcttccaggagcga-3', 5'-at gaccttgcccacagcctt-3'). PCR primers used for $\mathrm{C}_{\mu}$ germline (245 bp: 5'-tgggcaagtggatctgaaca-3', 5'-gaagacatttgggaagg actgact-3'), C $\gamma 1$ germline (429 bp: 5'-ggccttccagatcttgag$3^{\prime}, 5^{\prime}$-ggatccagagttccaggtcact-3') and $C_{\gamma} 1$ postswitch (353 bp: 5'-tgggcaagtggatctgaaca-3', 5'-ggatccagagttccaggtcact-3') transcripts were as described previously (Muramatsu et al., 2000). The PCR products were separated on agarose gels. The intensity of the bands was quantitated, and the normalized values were calculated based on the intensity of GAPDH bands.

\section{IB analysis}

After stimulation with anti-CD40 or transfection with expression plasmids, cell lysates were prepared in a $1 \%$ NP-40 lysis buffer containing protease and phosphatase inhibitors, as previously described (Lee and Koretzky, 1998). The lysates from $B$ lymphoma cells $\left(5 \times 10^{5}\right)$ and splenic $B$ cells $\left(1 \times 10^{6}\right)$ were mixed with $2 \times$ Laemmli sample buffer, boiled, and subjected to SDS-PAGE on $6 \%$ or $10 \%$ gels. After transferring proteins to a nitrocellulose membrane, IB was performed by blocking the membrane with $5 \%$ nonfat dried milk and incubating it with $\mathrm{Ab}$ against specific proteins, followed by incubation with an HRP-conjugated secondary $\mathrm{Ab}$. Chemiluminescence detection was conducted using enhanced chemiluminescence (ECL) reagents (Amersham Pharmacia Biotech Co., Arlington Heights, IL).

\section{Flow cytometry}

B lymphoma cells $\left(5 \times 10^{5}\right)$ and splenic B cells $\left(1 \times 10^{6}\right)$ were blocked with normal rabbit serum $(1: 1,000)$ in PBS followed by direct 2- or 3-color surface staining with various $\mathrm{Ab}$ combinations for $30 \mathrm{~min}$ on ice in staining buffer containing 1\% BSA in PBS. These cells, stained directly for surface molecules, were then fixed and permeabilized with a Cytofix/Cytoperm solution (BD PharMingen) for $20 \mathrm{~min}$ at $4^{\circ} \mathrm{C}$ and washed with a Perm/Wash solution (BD PharMingen). An Ab specific for p190RhoGEF was added, followed by incubation with an FITC- or allophycocyanin-conjugated secondary $\mathrm{Ab}$. Control staining was performed by incubating cells with FITC-, r-PE-, PerCP-, and allophycocyanin-conjugated anti-mouse IgGs. Stained cells were washed and resuspended in PBS. Data were immediately collected using a FACSCalibur and analyzed using CellQuest software (BD Biosciences, Mountain View, CA). For each experimental condition, 10,000 cells were analyzed. 
Fluorescence signals were analyzed as dot plots of the fluorescence intensity. The mean fluorescence intensities (MFIs) in the gated area, R, are also represented graphically in the figures.

\section{ELISA for Ig secretion}

Culture supernatants collected from fluorescent sorted stable cells $\left(2 \times 10^{6} / \mathrm{ml}\right)$ were serially diluted and added to 96-well Immulon-1 flat-bottomed plates that had been coated with anti-mouse $\operatorname{lgM}$ or IgG in carbonate-bicarbonate buffer (15 mM Na $2 \mathrm{CO}_{3}, 35 \mathrm{mM} \mathrm{NaHCO}$; pH 9.6) for 30 min at room temperature. The plates were treated with $3 \%$ bovine serum albumin blocking solution in PBS containing $0.05 \%$ Tween 20 (T-PBS). IgM or IgG was allowed to bind for $45 \mathrm{~min}$ at room temperature. Next, the plates were washed with T-PBS, and biotin-conjugated mouse $\operatorname{lgM}$ in conjunction with HRP-streptavidin or HRP-labeled mouse IgG-specific $A b$ was added to the wells for 30 min at room temperature. After the addition of the peroxidase substrate, o-phenylenediamine dihydrochloride, different $A b$ isotypes were quantified by measuring absorbance at $490 \mathrm{~nm}$ with a microplate reader (Molecular Devices, Spectra MAX 190).

\section{Other methods}

Protein concentrations were determined using the Bradford protein assay reagent (Bio-Rad) following the manufacturer's instructions. Comparisons between samples were performed using a two-tailed Student's $t$ test. Statistics were determined using Prism software (GraphPad Software, Inc.). Values of $P<0.05$ were considered significant.

\section{Acknowledgements}

We thank Drs. G. Bokoch, W. Pear, and G. Koretzky for providing valuable reagents. This study was supported by a grant of the Korea Healthcare technology R\&D Project, Ministry for Health, Welfare \& Family Affairs, Republic of Korea (A090743). Y.J.H., J.H.J., and Y.P. were supported in part by the second stage of the Brain Korea 21 Program of the Korea Ministry of Education.

\section{References}

Ahonen CL, Manning EM, Erickson LD, O'connor BP, Lind EF, Pullen SS, Kehry MR, Noelle RJ. The CD40-TRAF6 axis controls affinity maturation and the generation of long-lived plasma cells. Nat Immunol 2002;3:451-6

Arpin C, Dechanet J, Van Kooten C, Merville P, Grouard G, Briere F, Banchereau J, Liu YJ. Generation of memory B cells and plasma cells in vitro. Science 1995;268:720-2

Benson MJ, Erickson LD, Gleeson MW, Noelle RJ. Affinity of antigen encounter and other early B-cell signals determine B-cell fate. Curr Opin Immunol 2007;19:275-80

Boettner B, van Aelst L. The role of Rho GTPases in disease development. Gene 2002;286:155-74

Burridge K, Wennerberg K. Rho and Rac take centerstage.

\section{Cell 2004;116:167-79}

Calame KL. Plasma cells: finding new light at the end of B cell development. Nat Immunol 2001;2:1103-8

Delogu A, Schebesta A, Sun Q, Aschenbrenner K, Perlot T, Busslinger M. Gene repression by Pax 5 in B cells is essential for blood cell homeostasis and is reversed in plasma cells. Immunity 2006;24:269-81

Etienne-Manneville S, Hall A. Rho GTPases in cell biology. Nature 2002;420:629-35

Foy TM, Laman JD, Ledbetter JA, Aruffo A, Claassen E, Noelle RJ. gp39-CD40 interactions are essential for germinal center formation and the development of B cell memory. J Exp Med 1994;180:157-63

Gallagher E, Enzler T, Matsuzawa A, Anzelon-Mills A, Otero $D$, Holzer R, Janssen E, Gao M, Karin M. Kinase MEKK1 is required for CD40-dependent activation of the kinases Jnk and $\mathrm{p} 38$, germinal center formation, $\mathrm{B}$ cell proliferation and antibody production. Nat Immunol 2007;8:57-63

Gebbink MF, Kranenburg O, Poland M, van Horck FP, Houssa B, Moolenaar WH. Identification of a novel, putative Rho-specific GDP/GTP exchange factor and a RhoA-binding protein: control of neuronal morphology. J Cell Biol 1997; 137:1603-13

Gray D. A role for antigen in the maintenance of immunological memory. Nat Rev Immunol 2002;2:60-5

Gray D, Dullforce P, Jainandunsing S. Memory B cell development but not germinal center formation is impaired by in vivo blockade of CD40-CD40 ligand interaction. J Exp Med 1994;180:141-55

Gu Y, Filippi MD, Cancelas JA, Siefring JE, Williams EP, Jasti $A C$, Harris CE, Lee AW, Prabhakar R, Atkinson SJ, Kwiatkowski DJ, Williams DA. Hematopoietic cell regulation by Rac1 and Rac2 guanosine triphosphatases. Science 2003;302:445-9

Gu Y, Jasti AC, Jansen M, Siefring JE. RhoH, a hematopoietic-specific Rho GTPase, regulates proliferation, survival, migration, and engraftment of hematopoietic progenitor cells. Blood 2005;105:1467-75

Jabara H, Laouini D, Tsitsikov E, Mizoguchi E, Bhan A, Castigli E, Dedeoglu F, Pivniouk V, Brodeur S, Geha R. The binding site for TRAF2 and TRAF3 but not for TRAF6 is essential for CD40-mediated immunoglobulin class switching. Immunity 2002;17:265-76

Kallies A, Hasbold J, Fairfax K, Pridans C, Emslie D, McKenzie BS, Lew AM, Corcoran LM, Hodgkin PD, Tarlinton DM, Nutt SL. Initiation of plasma-cell differentiation is independent of the transcription factor Blimp-1. Immunity 2007;26:555-66

Kawabe T, Naka T, Yoshida K, Tanaka T, Fujiwara H, Suematsu S, Yoshida N, Kishimoto T, Kikutani H. The immune responses in CD40-deficient mice: impaired immunoglobulin class switching and germinal center formation. Immunity 1994;1:167-78

Klein U, Casola S, Cattoretti G, Shen Q, Lia M, Mo T, Ludwig T, Rajewsky K, Dalla-Favera R. Transcription factor IRF4 controls plasma cell differentiation and class-switch 
recombination. Nat Immunol 2006;7:773-82

Lee JR, Koretzky GA. Production of reactive oxygen intermediates following CD40 ligation correlates with c-Jun $\mathrm{N}$-terminal kinase activation and IL-6 secretion in murine $\mathrm{B}$ lymphocytes. Eur J Immunol 1998;28:4188-97

Lee JR, Ha YJ, Kim HJ. Cutting edge: Induced expression of a RhoA-specific guanine nucleotide exchange factor, p190RhoGEF, following CD40 stimulation and WEHI 231 B cell activation. J Immunol 2003;170:19-23

Li B, Yu H, Zheng W, Voll R, Na S, Roberts AW, Williams DA, Davis RJ, Ghosh S, Flavell RA. Role of the guanosine triphosphatase Rac2 in $\mathrm{T}$ helper 1 cell differentiation. Science 2000;288:2219-22

McHeyzer-Williams LJ, McHeyzer-Williams MG. Antigen-specific memory B cell development. Annu Rev Immunol 2005;23:487-513

Meili R, Firtel RA. Two poles and a compass. Cell 2003; 114:153-6

Meyer D, Liu A, Margolis B. Interaction of c-Jun amino-terminal kinase interacting protein-1 with p190 rhoGEF and its localization in differentiated neurons. J Biol Chem 1999; 274:35113-8

Muramatsu M, Kinoshita K, Fagarasan S, Yamada S, Shinkai Y, Honjo T. Class switch recombination and hypermutation require activation-induced cytidine deaminase (AID), a potential RNA editing enzyme. Cell 2000;102:553-63

Nera KP, Kohonen P, Narvi E, Peippo A, Mustonen L, Terho P, Koskela K, Buerstedde JM, Lassila O. Loss of Pax5 promotes plasma cell differentiation. Immunity 2006;24:283-93

Pear WS, Nolan GP, Scott ML, Baltimore D. Production of high-titer helper-free retroviruses by transient transfection. Proc Natl Acad Sci USA 1993;90:8392-6

Pear WS, Miller JP, Xu L, Pui JC, Soffer B, Quackenbush RC, Pendergast AM, Bronson R, Aster JC, Scott ML, Baltimore $D$. Efficient and rapid induction of a chronic myelogenous leukemia-like myeloproliferative disease in mice receiving P210 bcr/abl-transduced bone marrow. Blood 1998;92:3780-92

Pixley FJ, Xiong Y, Yu RY, Sahai EA, Stanley ER, Ye BH. BCL6 suppresses RhoA activity to alter macrophage morphology and motility. J Cell Sci 2005;118:1873-83

Qiang YW, Walsh K, Yao L, Kedei N, Blumberg PM, Rubin JS, Shaughnessy Jr J, Rudikoff S. Wnts induce migration and invasion of myeloma plasma cells. Blood 2005;106:1786-93

Randall TD, Heath AW, Santos-Argumedo L, Howard MC, Weissman IL, Lund FE. Arrest of B lymphocyte terminal differentiation by CD40 signaling: mechanism for lack of antibody-secreting cells in germinal centers. Immunity 1998;8:733-42

Reimold AM, Iwakoshi NN, Manis J, Vallabhajosyula P, Szomolanyi-Tsuda E, Gravallese EM, Friend D, Grusby MJ, Alt F, Glimcher LH. Plasma cell differentiation requires the transcription factor XBP-1. Nature 2001;412:300-7

Ridley AJ. Rho GTPases and cell migration. J Cell Sci 2001;114:2713-22

Rottner K, Hall A, Small JV. Interplay between Rac and Rho in the control of substrate dynamics. Curr Biol 1999;9:640-8

Saci A, Carpenter CL. RhoA GTPase regulates B cell receptor signaling. Mol Cell 2005;17:205-14

Sciammas R, Shaffer AL, Schatz JH, Zhao H, Staudt LM, Singh $\mathrm{H}$. Graded expression of interferon regulatory factor-4 coordinates isotype switching with plasma cell differentiation. Immunity 2006;25:225-36

Shaffer AL, Lin KI, Kuo TC, Yu X, Hurt EM, Rosenwald A, Giltnane JM, Yang L, Zhao H, Calame K, Staudt LM. Blimp-1 orchestrates plasma cell differentiation by extinguishing the mature B cell gene expression program. Immunity 2002; 17:51-62

Shaffer AL, Shapiro-Shelef M, Iwakoshi NN, Lee AH, Qian SB, Zhao H, Yu X, Yang L, Tan BK, Rosenwald A, Hurt EM, Petroulakis E, Sonenberg N, Yewdell JW, Calame K, Glimcher LH, Staudt LM. XBP1, downstream of Blimp-1, expands the secretory apparatus and other organelles, and increases protein synthesis in plasma cell differentiation. Immunity 2004;21:81-93

Shapiro-Shelef M, Calame K. Regulation of plasma-cell development. Nat Rev Immunol 2005;5:230-42

Shapiro-Shelef M, Lin KI, McHeyzer-Williams LJ, Liao J, McHeyzer-Williams MG, Calame K. Blimp-1 is required for the formation of immunoglobulin secreting plasma cells and pre-plasma memory B cells. Immunity 2003;19:607-20

Turner CA Jr, Mack DH, Davis MM. Blimp-1, a novel zinc finger-containing protein that can drive the maturation of $B$ lymphocytes into immunoglobulin-secreting cells. Cell 1994;77:297-306

van Horck FP, Ahmadian MR, Haeusler LC, Moolenaar WH, Kranenburg O. Characterization of p190RhoGEF, a RhoA-specific guanine nucleotide exchange factor that interacts with microtubules. J Biol Chem 2001;276:4948-56

Vicente-Manzanares M, Sanchez-Madrid F. Role of the cytoskeleton during leukocyte responses. Nat Rev Immunol 2004;4:110-22

Walmsley MJ, Ooi SK, Reynolds LF, Smith SH, Ruf S, Mathiot A, Vanes L, Williams DA, Cancro MP, Tybulewicz VL. Critical roles for Rac1 and Rac2 GTPases in B cell development and signaling. Science 2003;302:459-62

Xu J, Foy TM, Laman JD, Elliott EA, Waldschmidt TJ, Elsemore J, Noelle RJ, Flavell RA. Mice deficient for the CD40 ligand. Immunity 1994;1:423-31

Xu Z, Pone EJ, Al-Qahtani A, Park SR, Zan H, Casali P. Regulation of aicda expression and AID activity: relevance to somatic hypermutation and class switch DNA recombination. Crit Rev Immunol 2007;27:367-97

Ye BH, Cattoretti G, Shen Q, Zhang J, Hawe N, de Waard R, Leung C, Nouri-Shirazi M, Orazi A, Chaganti RS, Rothman P, Stall AM, Pandolfi PP, Dalla-Favera R. The BCL-6 proto-oncogene controls germinal-centre formation and Th2-type inflammation. Nat Genet 1997;16:161-70

Yoon SH, Cho HI, Kim TG. Activation of B cells using Schneider 2 cells expressing CD40 ligand for the enhancement of antigen presentation in vitro. Exp Mol Med 2005;37:567-74 